\title{
Decomposition of the optical transfer function: wavefront coding imaging systems
}

\author{
Gonzalo Muyo and Andy R. Harvey \\ School of Engineering and Physical Sciences, Heriot-Watt University, Riccarton, Edinburgh EH14 4AS, United \\ Kingdom
}

Received April 20, 2005; revised manuscript received June 27, 2005; accepted July 7, 2005

We describe the mapping of the optical transfer function (OTF) of an incoherent imaging system into a geometrical representation. We show that for defocused traditional and wavefront-coded systems the OTF can be represented as a generalized Cornu spiral. This representation provides a physical insight into the way in which wavefront coding can increase the depth of field of an imaging system and permits analytical quantification of salient OTF parameters, such as the depth of focus, the location of nulls, and amplitude and phase modulation of the wavefront-coding OTF. (C) 2005 Optical Society of America

OCIS codes: $080.3620,110.0110,110.4850$.

The optical transfer function (OTF) is a critical parameter of aberrated optical systems. Except in restricted cases, such as when the well-known Fouriertransform relationships are valid, ${ }^{1}$ the numerical methods of wave-optics analysis can obscure the underlying physical processes of OTF formation. We show here how a geometrical analysis of a phasor representation of a decomposed OTF enables an improved description and quantification of OTF parameters to be made. Although this approach is pertinent to all aspects of incoherent image formation, its application to wavefront coding ${ }^{2}$ (WC) is of particular interest. When it is combined with digital postprocessing, a WC mask placed in the pupil plane of a conventional imager produces greatly reduced sensitivity to defocus-related optical aberrations. ${ }^{2-4}$ The resultant OTFs are approximately invariant over a restricted range of defocus, but there has been no reported analytical treatment of the range of defocus invariance, of the magnitude of departures from invariance, or of a physical, wave-optics explanation of the underlying physical processes. In this Letter we demonstrate that the OTF of a defocused WC system can be considered in terms of a generalized Cornu spiral (GCS) and that the performance parameters of a WC system can be readily derived from the geometry of such a spiral. We start by describing how the OTF can be decomposed to enable its composition to be plotted as a curve in the complex plane. We then apply this technique to an imaging system, incorporating first defocus and then both defocus and a cubic phase function.

Consider first the decomposition of the OTF: For clarity the analysis is restricted to the case of a onedimensional optical system. The OTF, $L(f)$, for spatially incoherent illumination is determined by the normalized autocorrelation of the pupil function, $P(x)$ :

$$
\begin{aligned}
L(f)= & \int_{-\infty}^{\infty} P\left(x+R \frac{f}{f_{\max }}\right) \\
& \times P^{*}\left(x-R \frac{f}{f_{\max }}\right) \mathrm{d} x / \int_{-\infty}^{\infty}|P(x)|^{2} \mathrm{~d} x,
\end{aligned}
$$

where $x$ is the transverse linear coordinate, $R f / f_{\max }$ is a pupil-plane coordinate representing spatial frequency $f, R$ is the transverse half-width of the pupil, and $f_{\max }$ is the cutoff spatial frequency. We define the integral

$$
H\left(r^{\prime}, v\right)=\int_{0}^{r^{\prime}} h(r, v) \mathrm{d} r / \int_{-1}^{1} h(r, 0) \mathrm{d} r,
$$

with $\quad h(r, v)=P(r+v) P^{*}(r-v) \quad$ and $\quad h(r, 0)=|P(r)|^{2}$, where $r=x / R$ is the normalized pupil coordinate and $v=f / f_{\max }$ is the normalized spatial frequency. The normalized OTF is $L(v)=H(1-v, v)-H(-1+v, v)$. We now decompose the OTF, $L(f)$, into phasor components, $h(r, v)$. We represent incremental contributions $h \mathrm{~d} r$ in the complex plane and perform the integral in Eq. (2) as $r^{\prime}$ is varied from zero to the negative limit, $-1+v$, and from zero to the positive limit, $1-v$.

As an illustration of the usefulness of this approach, and before considering its application to WC, we consider first the decomposition of the OTF of an optical system that suffers only from defocus, which we represent by a quadratic phase factor. The pupil function is $P\left(r ; w_{20}\right)=p(r) \exp \left(i 2 \pi w_{20} r^{2}\right)$, where $w_{20}$ is the defocus coefficient in units of wavelength. To simplify the description we assume that $|P(r)|=1$. The phasor representation in this case is given by

$$
h\left(r, v ; w_{20}\right)=\exp \left(i 8 \pi w_{20} v r\right),
$$

so the phasor contributions to $L\left(v ; w_{20}\right)$ have a phase that varies linearly with offset $r^{\prime}$; that is, $H\left(r^{\prime}, v ; w_{20}\right)$ describes the arc of a circle traced from the origin in the complex plane. The defocused OTF, $L\left(v ; w_{20}\right)$ $=H\left(1-v, v ; w_{20}\right)-H\left(-1+v, v ; w_{20}\right)$, is the vector displacement between the ends of this arc. The Hermitian nature of $h\left(r, v ; w_{20}\right)$ means that the integral represented by $H\left(r^{\prime}, v ; w_{20}\right)$ is always real. The variation of $L\left(v ; w_{20}\right), H\left(1-v, v ; w_{20}\right)$ and $H\left(-1+v, v ; w_{20}\right)$, is illustrated in Figs. 1(a) $-1(\mathrm{e})$ for $v=0.5$ and for a range of values of $w_{20}$. For a specific value of $v$, nulls occur in the defocused OTF when the gradient of the phasor at both limits $r^{\prime}=1-v$ and $r^{\prime}=-1+v$ is zero; that is, the phasors $h\left[ \pm(1-v), v ; w_{20}\right]=\exp (i n \pi)$, where $n$ is a nonzero positive integer. This enables us to write $w_{20}= \pm n / 8 v(1-v)$ for the values of defocus that intro- 


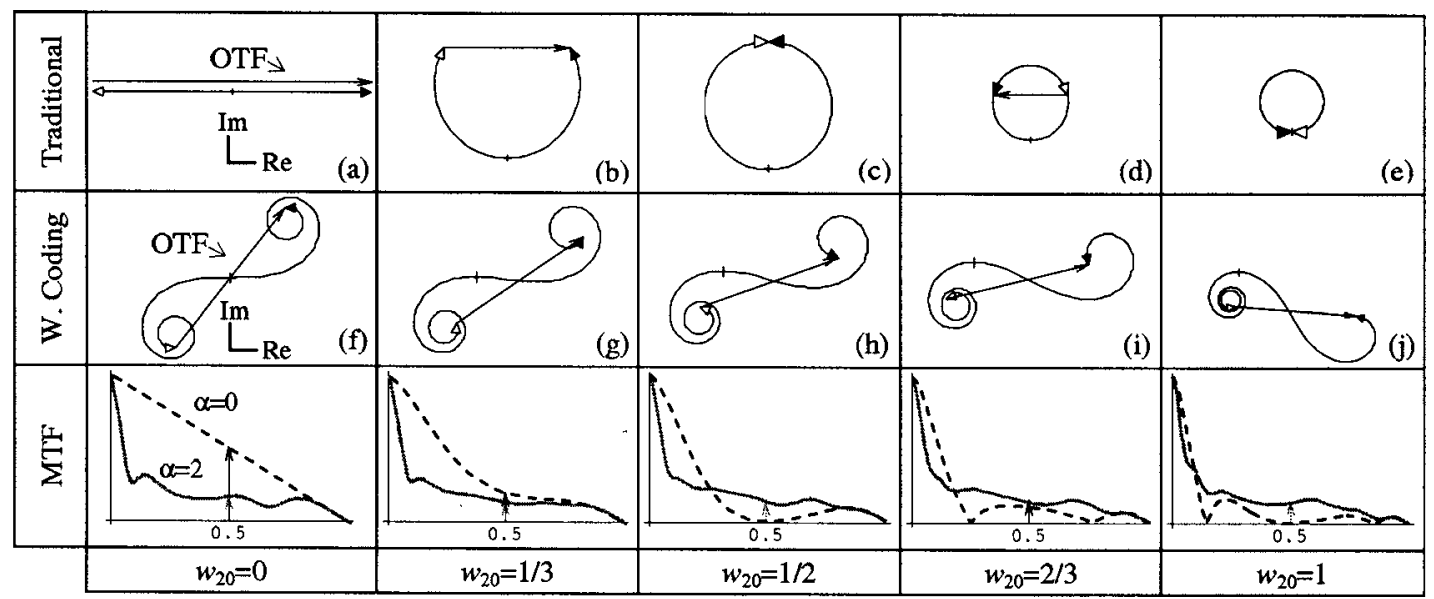

Fig. 1. (a)-(e) Traditional defocused OTFs and MTFs depicted as arc circles for $\alpha=0$ and $v=0.5$. (f) $-(\mathrm{j}$ ) WC OTFs and MTFs depicted as GCSs for $\alpha=2$ and $v=0.5$. Note the absence of both nulls and phase reversal for the WC MTFs (solid curves), in contrast to the defocused OTFs (dashed curves).

duce zeros into the OTF. It can be appreciated from Figs. 1(a)-1(e) that, when the pupil function is even, the magnitude of $L\left(v ; w_{20}\right)$ decreases rapidly with added defocus, and, for $w_{20} \geqslant 0.5$, nulls and phase reversals are introduced. In this case, decomposition of the OTF into its constituent phasors enables a simple and elegant explanation of the location of the zeros to be made, a result that can also be found in optics textbooks, such as Ref. 6.

We now show how this decomposition of the OTF provides a simple explanation and parameterization of the WC process. We consider a one-dimensional, defocused optical system in which a cubic phase mask with peak-to-valley displacement $2 \alpha$, in units of wavelengths, is located in the pupil. The generalized pupil function for this system is

$$
P\left(r, v ; w_{20}, \alpha\right)=p(r) \exp \left[i 2 \pi\left(w_{20} r^{2}+\alpha r^{3}\right)\right],
$$

with $p(r)=1$ for $|r| \leqslant 1$ and $p(r)=0$ for $|r|>1$. The interferogram phasors are thus given by

$$
h\left(r, v ; w_{20}, \alpha\right)=\exp \left[i 4 \pi v\left(3 \alpha r^{2}+2 w_{20} r+\alpha v^{2}\right)\right] .
$$

The complex decomposition of the OTF is obtained by inserting Eq. (5) into Eq. (2) and plotting the variation of $H\left(r^{\prime}, v ; w_{20}, \alpha\right)$ as $r^{\prime}$ varies from $-1+v$ to $1-v$ :

$$
\begin{aligned}
H\left(r^{\prime}, v ; w_{20}, \alpha\right)= & \frac{1}{2} \int_{0}^{r^{\prime}} \exp \left[i 4 \pi v \left(3 \alpha r^{2}+2 w_{20} r\right.\right. \\
& \left.\left.+\alpha v^{2}\right)\right] \mathrm{d} r .
\end{aligned}
$$

This integral describes arc-length parameterized curves known as GCSs, or clothoids. ${ }^{7}$ Although an analytical expression for $H\left(r^{\prime}, v ; w_{20}, \alpha\right)$ and $L\left(v ; w_{20}, \alpha\right)$ can be obtained in terms of error functions, a physical understanding and analysis of the WC process is more readily obtained from consideration of the simpler expression of Eq. (5). The three terms in the exponential contribute to a compound curve in the complex plane with three distinct components: $\alpha v^{2}$ yields a linear section; $3 \alpha r^{2}$ induces a diminishing clockwise spiral for both positive and negative pupil displacements; and, when $w_{20}$ is sufficiently large, $2 w_{20} r$ introduces arcs of a circle similar to normal defocus as described above. Consequently, when $w_{20}$ is sufficiently small the compound curve consists of two clockwise spirals separated by a linear section.

$H\left(r^{\prime}, v ; w_{20}, \alpha\right)$ is plotted in Figs. 1(f) $-1(\mathrm{j})$ for $\alpha=2$ and as $w_{20}$ is varied from 0 to 1 for $v=0.5$. Also shown are the corresponding modulation-transfer functions (MTFs) for this system. The resultant OTF, $L\left(v ; w_{20}, \alpha\right)$, is indicated by the resultant phasor between the inner ends of the two spirals. It can be seen that increasing positive defocus causes the spiral structure to rotate clockwise about the origin such that one spiral becomes more tightly wound while the opposite spiral unwinds such that, in terms of the MTF, $\left|L\left(v ; w_{20}, \alpha\right)\right|$, the effect of one approximately counteracts the other. For negative defocus the rotation is also clockwise but produces tightening and loosening of the coils about the foci opposite those of positive defocus. In consequence, $\left|L\left(v ; w_{20}, \alpha\right)\right|$ remains approximately constant until one spiral is completely wound around the origin and the other spiral is completely unwound; as $\left|w_{20}\right|$ increases beyond this point, $\left|L\left(v ; w_{20}, \alpha\right)\right|$ decreases rapidly. Physically, the coiling for positive and negative $r^{\prime}$ represents the result of the combined effects of the cubic phase mask and the defocus; tightening in the spirals occurs when the cubic phase mask introduces phasefront curvature that is in the same sense as the quadratic defocus, and loosening of the spirals occurs when the cubic phase mask tends to cancel the quadratic defocus. It can be appreciated from this picture how reduced sensitivity to defocus is facilitated by an odd pupil function.

Having shown how decomposition of the OTF enables WC to be described as a GCS, we now show how we can use the geometry of the GCS to estimate important parameters such as (1) an approximation of the value for $\left|L\left(v ; w_{20}, \alpha\right)\right|,(2)$ the maximum value of $w_{20}$ for which the MTF can be considered to be approximately constant, (3) the magnitude of amplitude 
modulation of $\left|L\left(v ; w_{20}, \alpha\right)\right|$ within this invariance region, and (4) the magnitude of the phase modulation introduced. Previous descriptions of WC did not provide any measure of the second of these parameters and predicted that the last two will be equal to zero.

The spatial-frequency integral in Eq. (6) converges rapidly to the foci of the spiral with increasing pupilplane displacement $r^{\prime}$, and so an approximation of the mean value of the OTF can be calculated by taking the limit $r^{\prime} \rightarrow \pm \infty$ in $H\left(r^{\prime}, v ; w_{20}, \alpha\right)$, which yields

$$
\left\langle L\left(v ; w_{20}, \alpha\right)\right\rangle \approx \frac{(1+i)}{4 \sqrt{6 \alpha v}} \exp \left[-i 4 \pi v\left(-\alpha v^{2}+\frac{w_{20}^{2}}{3 \alpha}\right)\right] .
$$

The GCS rotates clockwise as $w_{20}$ is increased in both positive and negative directions. In the ray picture, this phase variation of the OTF represents the parabolic shape of the caustic along which the center of the point-spread function moves with varying defocus. ${ }^{3}$ The mean MTF is calculated by taking the modulus of the previous expression; $\left|\left\langle L\left(v ; w_{20}, \alpha\right)\right\rangle\right|$ $=1 / \sqrt{48 \alpha v}$. This expression is valid for moderate values of $\alpha$ and spatial frequencies $0<v<1$.

When one end of the spiral is completely unwrapped, increasing defocus causes the MTF to drop rapidly. We define the maximum value for defocus invariance as that value of $w_{20}$ for which the spiral is sufficiently unwrapped that the end coincides with the point of inflection midway between the two foci of the spiral, that is, the point at which the MTF is reduced to $50 \%$ of the mean value. Given that curvature $\kappa$, at displacement $r^{\prime}$ on a GCS, can be written as

$$
\kappa\left(r^{\prime}, v ; w_{20}, \alpha\right)=16 \pi v\left(w_{20}+3 \alpha r^{\prime}\right),
$$

setting $\kappa=0$ to denote the point of inflection, and substituting $r^{\prime}=1-v$ or $r^{\prime}=-1+v$, we obtain the maximum defocus for an invariant MTF:

$$
\left|w_{20}\right|_{\max }=3 \alpha(1-v) \text {. }
$$

The representation in Figs. 1(f)-1(j) shows the OTF vector between the ends of the spiral oscillating in amplitude and phase about the mean OTF vector joining the foci of the spiral; the OTF vector clearly represents oscillatory amplitude and phase modulation of the OTF that accompanies varying defocus. These modulations can be quantified from the geometry of the GCS. Projection of the displacement vectors between each focus (i.e., for $r^{\prime}= \pm \infty$ ) and the respective end of the spiral at $r^{\prime}= \pm(1-v)$ onto the mean OTF vector yields amplitude modulation $M_{\|}$, whereas projection in the orthogonal direction $M_{\perp}$ yields phase modulation $\Delta \theta$. It can thus be shown that the amplitude modulation is given by

$$
\begin{aligned}
& M_{\|}\left(v ; w_{20}, \alpha\right) \\
& \quad=\frac{1}{16 \pi v}\left(\frac{\cos \left\{(4 \pi v / 3 \alpha)\left[w_{20}-3 \alpha(1-v)\right]^{2}+(\pi / 4)\right\}}{w_{20}-3 \alpha(1-v)}\right. \\
& \left.\quad-\frac{\cos \left\{(4 \pi v / 3 \alpha)\left[w_{20}+3 \alpha(1-v)\right]^{2}+(\pi / 4)\right\}}{w_{20}+3 \alpha(1-\nu)}\right),
\end{aligned}
$$

where each of the two components within the boldface parentheses corresponds to modulation at either end of the spiral.

The modulation $M_{\perp}$ is readily obtained by replacing the cosine with a sine function in Eq. (10), and for $w_{20}>0$ we obtain the amplitude of the phase modulation:

$$
\begin{aligned}
\Delta \theta= & \frac{\sqrt{3 \alpha v}}{2 \pi v\left[w_{20}-3 \alpha(1-v)\right]} \\
& \times \sin \left\{\frac{4 \pi v}{3 \alpha}\left[w_{20}-3 \alpha(1-v)\right]^{2}+\frac{\pi}{4}\right\} .
\end{aligned}
$$

In summary, we have developed a new understanding of the formation of the OTF in incoherent imaging systems that involves a geometrical representation of the contributing complex interferogram components. This provides a lucid quantification of the salient parameters of the OTF both for a defocused traditional imaging system and for a defocused system incorporating a WC element. Features that are evident in the OTFs of wavefront-coded systems, such as amplitude modulation and the absence of nulls, have been explained analytically for the first time to our knowledge; thus the possibility of simple calibration has been introduced. It is noteworthy that the two-dimensional integral of the ambiguity function, as employed in Ref. 1 to synthesize the phase masks, can be effectively simplified to the equation for a straight line [Eq. (8)].

This study was carried out with funding from QinetiQ Ltd., Malvern, UK. A. Harvey's email address is a.r.harvey@hw.ac.uk.

\section{References}

1. S. Mezouari and A. R. Harvey, J. Opt. A Pure Appl. Opt. 5, S86 (2003).

2. E. Dowski and T. W. Cathey, Appl. Opt. 34, 1859 (1995).

3. W. T. Cathey and E. R. Dowski, Appl. Opt. 41, 6080 (2002).

4. S. Mezouari and A. R. Harvey, Opt. Lett. 28, 771 (2003).

5. M. Born and E. Wolf, Principles of Optics, 7th ed. (Cambridge U. Press, 1999), p. 548.

6. J. W. Goodman, Introduction to Fourier Optics, 2nd ed. (McGraw-Hill, 1996).

7. J. M. Ali, R. M. Tookey, J. V. Ball, and A. A. Ball, J. Comput. Appl. Math. 102, 37 (1999). 